\title{
Role of Eosinophilic Inflammation in Disease Severity among Egyptian COVID-19 Patients
}

\author{
Mariam F. Abdelmaksoud ${ }^{1}$, Shaimaa H. Fouad ${ }^{2}$, Hieba G. Ezzelregal ${ }^{3}$, \\ Shereen A. Baioumy ${ }^{4}$, and Sara I. Taha ${ }^{1}$ \\ ${ }^{1}$ Department of Clinical Pathology, Faculty of Medicine, Ain Shams \\ University, Cairo, Egypt. \\ ${ }^{2}$ Department of Internal Medicine/Allergy and Clinical Immunology, Faculty of \\ Medicine, Ain Shams University, Cairo, Egypt. \\ ${ }^{3}$ Department of Pulmonary Medicine, Faculty of Medicine, Ain Shams University, \\ Cairo, Egypt. \\ ${ }^{4}$ Department of Microbiology and Immunology, Faculty of Medicine, Zagazig \\ University, Zagazig, Egypt.
}

Corresponding Author Sara Ibrahim Taha

Mobile:

$+(20) 1125360009$

E mail:

dr_sara_ib@med.asu.e du.eg

Key words: COVID-19;

Eosinophils; Lymphopenia; Severity; Total leucocytic count.
Background and study aim: The coronavirus disease pandemic of 2019 (COVID-19) has created global health and economic implications. All potential biomarkers, risk factors, therapy and preventative measures of the disease has been thoroughly investigated. This study examined the relationship between eosinophils and COVID-19 severity, as well as other clinical and laboratory markers of the disease.

Patients and Methods: In this retrospective study, we collected data from 162 patients' medical records including baseline complete blood counts with differential total leucocytic counts (TLC). Descriptive and comparative statistics were performed.

\section{INTRODUCTION}

The novel coronavirus, Severe Acute Respiratory Syndrome Coronavirus 2 (SARS-CoV-2), is unprecedentedly causing the worldwide coronavirus disease 2019 (COVID-19) pandemic. All efforts are directed towards the understanding of its diagnostic biomarkers, risk factors, and novel strategies for its prevention and treatment [1].

Eosinophils arise from pluripotent progenitor cells in the bone marrow under the control of granulocytemonocyte colony-stimulating factor (GMCSF), interleukin (IL)-3, and IL5 [2]. They constitute a small portion of leukocytic pool in the blood. After
Results: Regarding disease severity, TLC significantly increased $(\mathrm{p}=0.019)$ and lymphocyte count significantly decreased $(\mathrm{p}=0.003)$ with more severe disease but eosinophil count showed no significant differences $(p=0.864)$. Patients admitted to the ICU showed no significant difference in eosinophil count $(\mathrm{p}=0.551)$, they had significantly higher TLC $(\mathrm{p} \leq 0.001)$ and significantly lower lymphocyte count $(\mathrm{p} \leq 0.001)$. No significant correlations $(\mathrm{p}>0.05)$ were found between eosinophil count and any of the laboratory markers of the disease, age of patients, or length of hospital stay.

Conclusion: Eosinophil count had no correlation with COVD-19 severity, while lymphopenia was a poor prognostic marker.

being released to the circulation, they migrate to tissues. They are commonly increased in association with parasitic and allergic conditions to deliver inflammatory mediators like (histamine, leukotrienes, plateletactivating factor, adenosine, and bradykinin) to promote host defense, which is accompanied by collateral tissue damage [3]. The US National Institutes of Health Clinical Center Laboratory's normal range for blood eosinophils is $40-360$ cells per microliter or $0.7-5.8 \%$ of the total leukocytic count. Eosinophilia is defined as > 500 eosinophils per microliter of blood, while eosinopenia is $<10$ eosinophils per microliter of blood [4]. 
The role of eosinophils in COVID-19 infection is being investigated. Eosinopenia, together with lymphopenia, were identified as diagnostic markers in COVID-19 [5]. Moreover, eosinopenia was correlated with severity and poor outcome. However, this finding is neither definitive nor pathognomonic for COVID-19 [4].

This study aimed to determine the relationship between eosinophils and COVID-19 severity as well as the other clinical and laboratory markers in COVID-19 patients.

\section{MATERIALS AND METHODS}

\section{Study subjects and settings}

A total of 162 adult patients (age $\geq 18$ years) recruited from COVID-19 isolation hospitals of Ain Shams University from September to November 2020 were enrolled in this study. Of them, only 159 patients were positive for SARS$\mathrm{CoV}-2$ by initial reverse transcriptionpolymerase chain reaction (RT-PCR) testing for nasal and pharyngeal swab specimens. Patients with aplastic anemia, lymphoproliferative disorders, immune deficiency disorders, or drug intake history that affects eosinophil counts like epinephrine, thyroxin, or corticosteroids were excluded. Ain Shams University Faculty of Medicine Research Ethics Committee (REC) approved this study.

In this retrospective study we collected data from patients' medical records, including age, gender, clinical presentations at the time of hospital admission, associated co-morbidities, duration of hospital stay, the requirement for ICU admission, and medications received.

Baseline routine laboratory data including complete blood count (CBC) parameters with differential white blood cell count, C-reactive protein (CRP), D-dimer, and ferritin that were tested according to the standard automated methods at Ain Shams Hospitals' Central Laboratories as well as findings of chest CT that were performed in the Radiology Department were also collected from the patients' hospital files. Patients were diagnosed and divided into mild, moderate, and severe groups based on the WHO interim guidance [6]. The mild and moderate groups had clinical signs of pneumonia (fever, cough, anorexia, malaise, and muscle pain). The severe group had respiratory distress, respiratory rate $>30$ breaths/min in resting state, mean oxygen saturation $<93 \%$, and arterial blood oxygen partial pressure $(\mathrm{PaO} 2) /$ oxygen concentration (FiO 2) $\leq 300 \mathrm{mmHg}$ [ [6].

\section{COVID-19 Reporting and Data System (CO- RADS):}

According to the clinical findings and laboratory test results of patients, as well as CT records, COVID-19 infection suspicion level is graded by CO-RADS score. While CO-RADS 0 indicates negative infection, COVID-19 infection suspicion in CO-RADS 1: is highly unlikely, the CT is normal with findings of non-infectious disease; CO-RADS 2: is low, consistent CT findings with other infections; CO-RADS 3: is indeterminate, CT abnormalities indicate infection but are not specifically sure of COVID19; CO-RADS 4: is high, most CT findings are suspicious but not extremely typical as multifocal consolidations, unilateral ground glass, or confluent; and CO-RADS 5: is high with typical CT findings; and CO-RADS 6 reflects positive RT-PCR SARS-Cov2 infection [7].

\section{Statistical analysis:}

Medians and interquartile ranges were used to describe quantitative variables, while qualitative variables were described as frequencies and percentages. Comparisons were made using the Mann-Whitney U test, and the Kruskal-Wallis test was used. For the assessment of non-random associations between qualitative variables, Chisquared or Fisher exact test was performed. Spearman's coefficients represented correlations. The statistical analyses were performed using SPSS version 20.0 software, a p-value $<0.05$ was considered statistically significant.

\section{RESULTS:}

This study included 162 patients, 76 (46.9\%) were male and $86(53.1 \%)$ were female. The average age was $47.51 \pm 16.30$ years. According to disease severity at the time of admission, 68 $(42.0 \%)$ patients were mild, $69(42.6 \%)$ were moderate and $25(15.4 \%)$ were severe. Hypertension (28.4\%) and diabetes (17.9\%) were the most common comorbidities. Only $11(6.8 \%)$ patients were smokers. The most common complaints during ER visits were fever (40.1\%) and dyspnea $(27.2 \%)$, while $11(6.8 \%)$ of all patients had no symptoms at all. Most of the patients (total number $120 ; 74.1 \%$ ) reported a 
previous contact with a confirmed case of COVID-19. Of all studied subjects, 45 (27.8\%) required ICU admission and only 10 (6.2\%) died. The median hospitalization duration was 10 (IQR: 6 - 13) days. The baseline characteristics of the included patients are shown in Table 1.

Comparisons made according to total leucocytic count (TLC), lymphocyte and eosinophil counts of patients included in the study, revealed no significant difference in sex $(\mathrm{p}=0.585,0.518$ and 0.607 , respectively) (Tables 2a,3a,4a). Regarding co-morbidities, the diabetic patients had statistically significant higher TLC compared to the non-diabetics (median: 8.00, (IQR: $6.90-$ $13.00) \times 10^{3} / \mathrm{cmm} \quad$ vs. $6.30 \quad(4.79 \quad-\quad 8.00)$ $\left.\times 10^{3} / \mathrm{cmm} ; \mathrm{p}=0.002\right)$. In addition, patients with chronic liver disease (CLD) had statistically significant higher TLC (median: 25.00; (IQR: $13.00-27.00) \times 10^{3} / \mathrm{cmm}$ vs. $6.70(4.80-8.81)$ $\left.\times 10^{3} / \mathrm{cmm} ; \mathrm{p}=0.008\right)$ (Table $2 \mathrm{~b}$ ) and statistically significant lower lymphocyte count (median: 0.64 (IQR: $0.40-0.70) \times 10^{3} / \mathrm{cmm}$ vs. $1.50(0.80$ - 2.18) $\times 10^{3} / \mathrm{cmm} ; \mathrm{p}=0.028$ ) compared to those with no CLD. Also, hypertensive patients had statistically significant lower lymphocyte count compared to the non-hypertensives (median: 1.14 (IQR: $0.70-1.92) \times 10^{3} / \mathrm{cmm}$ vs. $1.60(0.90-$ 2.24) $\times 10^{3} / \mathrm{cmm} ; \mathrm{p}=0.040$ ) (Table $3 \mathrm{~b}$ ). On the other hand, eosinophil count did not show significant changes with any of the comorbidities ( $p>0.05)$ (Table 4b).

Regarding disease severity, TLC was significantly increased $(\mathrm{p}=0.019)$ (Table $2 \mathrm{a})$ and lymphocyte count significantly decreased $(\mathrm{p}=0.003)$ (Table 3a) with more severe disease but eosinophil count did not show any significant differences $(\mathrm{p}=0.864)$ (Table 4a). Median (IQR) TLC was $5.80(4.80-7.67) \times 10^{3} / \mathrm{cmm}$ in mild, $6.90(4.99-9.30) \times 10^{3} / \mathrm{cmm}$ in moderate and $9.00(4.80-17.00) \times 10^{3} / \mathrm{cmm}$ in severe patients, while median (IQR) lymphocyte count was 1.78 $(1.28-2.54) \times 10^{3} / \mathrm{cmm}$ in mild, $1.17(0.70-$ $2.00) \times 10^{3} / \mathrm{cmm}$ in moderate and $1.15(0.64-$ $1.92) \times 103 / \mathrm{cmm}$ in severe patients.

By investigating the effect of drug intake on blood cell count we found that, eosinophil count was significantly increased in patients treated with Clexane (median: $0.04 \quad(0.00-0.07)$ $\times 10^{3} / \mathrm{cmm}$ vs. $0.00(0.00-0.00) \times 10^{3} / \mathrm{cmm}$; $\mathrm{p}=0.039$ ) (Table 4c). On the other hand, TLC significantly decreased in patients treated with Plaquenil (median: 6.34 (IQR: 4.80 - 8.00) $\times 10^{3} / \mathrm{cmm}$ vs. $8.00(5.90-15.20) \times 10^{3} / \mathrm{cmm}$; $\mathrm{p}=0.002)$, and significantly increased in patients treated with steroids (median: 7.10 (IQR: $4.81-$ $10.11) \times 10^{3} / \mathrm{cmm} \quad$ vs. $5.80 \quad(4.80 \quad-7.67)$ $\times 10^{3} / \mathrm{cmm} ; \quad \mathrm{p}=0.039$ ) (Table 2c). Besides, lymphocyte count was significantly decreased by intake of Actemra (median: 1.01 (IQR: $0.74-$ $1.30) \times 10^{3} / \mathrm{cmm}$ vs. $1.58(0.86-2.30) \times 10^{3} / \mathrm{cmm}$; $\mathrm{p}=0.014)$ and steroids (median: 1.17 (IQR: $0.70-$ $2.00) \times 10^{3} / \mathrm{cmm} \quad$ vs. $1.78 \quad(1.28 \quad-2.54)$ $\left.\times 10^{3} / \mathrm{cmm} ; \mathrm{p}=0.001\right)($ Table $3 \mathrm{c})$.

Patients admitted to the ICU showed no significant difference in eosinophil count (median: 0.04 (IQR: $0.00-0.07$ ) $\times 10^{3} / \mathrm{cmm}$ vs. $\left.0.03(0.00-0.07) \times 10^{3} / \mathrm{cmm} ; \mathrm{p}=0.551\right)$ (Table 4a), they had significantly higher TLC (median: 8.00 (IQR: $6.00-16.70) \times 10^{3} / \mathrm{cmm}$ vs. 6.00 $\left.(4.80-7.90) \times 10^{3} / \mathrm{cmm} ; \mathrm{p} \leq 0.001\right)($ Table $2 \mathrm{a})$ and significantly lower lymphocyte count (median: 0.80 (IQR $0.58-1.60) \times 10^{3} / \mathrm{cmm}$ vs. $\left.1.70(1.14-2.34) \times 10^{3} / \mathrm{cmm} ; \mathrm{p} \leq 0.001\right)($ Table 3a) when compared to patients who did not require ICU admission and were treated at the hospital ward. None of the TLC, lymphocyte and eosinophil counts has changed significantly according to the patients' outcome $(\mathrm{p}=0.125$, 0.997 and 0.994 , respectively).

In all included patients, TLC showed significant positive correlations with COVID-19 CO-RADS classification $(\mathrm{rs}=0.161 ; \mathrm{p}=0.041)$, platelet count $(\mathrm{rs}=0.211 ; \mathrm{p}=0.007), \quad \mathrm{CRP} \quad(\mathrm{rs}=0.324 ; \mathrm{p} \leq$ $0.001)$, ferritin $(\mathrm{rs}=0.232 ; \mathrm{p}=0.003)$ and $\mathrm{d}$-dimer levels ( $\mathrm{rs}=0.279 ; \mathrm{p} \leq 0.001$ ). In addition, Lymphocyte count showed a significant positive correlation with hemoglobin level $(\mathrm{rs}=0.331 ; \mathrm{p} \leq$ 0.001 ) and significant negative correlations with age ( $\mathrm{rs}=-0.187 ; \mathrm{p}=0.019)$, COVID-19 CORADS classification ( $r s=-0.198 ; p=0.013$ ), CRP $(\mathrm{rs}=-0.218 ; \mathrm{p}=0.006)$, ferritin $(\mathrm{rs}=-0.314 ; \mathrm{p} \leq$ $0.001)$ and d-dimer levels ( $r s=-0.260 ; p=0.001)$. In contrast, there were no significant correlations ( $p>0.05$ ) between eosinophil count and any laboratory test results, age of patients, COVID19 CO-RADS classification or length of hospital stay. Table 5 
Table (1): Baseline characteristics of the included COVID-19 patients ( $n=162)$.

\begin{tabular}{|c|c|c|}
\hline \multicolumn{3}{|c|}{ Total $(n=162)$} \\
\hline & Age (years) & $\begin{array}{c}\text { Mean } \pm \text { SD: } 47.51 \pm 16.30 \\
\text { Range: }(17-86)\end{array}$ \\
\hline Demographics & Sex & $\begin{array}{c}\text { Female: } 86(53.1 \%) \\
\text { Male } 76(46.9 \%)\end{array}$ \\
\hline Source of infection & $\begin{array}{l}\text { Unknown } \\
\text { Contact }\end{array}$ & $\begin{array}{c}42(25.9 \%) \\
120(74.1 \%)\end{array}$ \\
\hline Comorbidities & $\begin{array}{c}\text { SMOKING } \\
\text { DM } \\
\text { HTN } \\
\text { CLD } \\
\text { CKD } \\
\text { On dialysis } \\
\end{array}$ & $\begin{array}{c}1(6.8 \%) \\
29(17.9 \%) \\
46(28.4 \%) \\
3(1.9 \%) \\
12(7.5 \%) \\
2(1.2 \%) \\
\end{array}$ \\
\hline CO-RADS & $\begin{array}{c}\text { Median (IQR) } \\
\text { Range }\end{array}$ & $\begin{array}{c}4(1-4) \\
1-5 \\
\end{array}$ \\
\hline Severity & $\begin{array}{c}\text { Mild } \\
\text { Moderate } \\
\text { Severe } \\
\end{array}$ & $\begin{array}{l}68(42.0 \%) \\
69(42.6 \%) \\
25(15.4 \%)\end{array}$ \\
\hline Symptoms & $\begin{array}{c}\text { Asymptomatic } \\
\text { Fever } \\
\text { Cough } \\
\text { Diarrhea } \\
\text { Dyspnea } \\
\text { Fever + Diarrhea } \\
\text { Fever + Cough } \\
\text { Fever + Cough + Dyspnea } \\
\text { Fever + Dyspnea } \\
\text { Cough + Diarrhea } \\
\text { Fever + Bony aches }\end{array}$ & $\begin{array}{c}11(6.8 \%) \\
65(40.1 \%) \\
11(6.8 \%) \\
7(4.3 \%) \\
44(27.2 \%) \\
5(3.1 \%) \\
2(1.2 \%) \\
13(8.0 \%) \\
2(1.2 \%) \\
1(0.6 \%) \\
1(0.6 \%)\end{array}$ \\
\hline Drug therapy & $\begin{array}{c}\text { Plaquenil } \\
\text { Zithro } \\
\text { Clexane } \\
\text { Tamiflu } \\
\text { Avigan } \\
\text { Remdesivir } \\
\text { Steroids } \\
\text { Actemra } \\
\end{array}$ & $\begin{array}{c}132(82.0 \%) \\
162(100.0 \%) \\
153(94.4 \%) \\
34(21.0 \%) \\
1(0.6 \%) \\
4(2.5 \%) \\
94(58.0 \%) \\
19(11.7 \%) \\
\end{array}$ \\
\hline Laboratory & $\begin{array}{c}\text { Initial PCR } \\
\text { HB gm/dl } \\
\text { PLT } \times 10^{3} / \mu \mathrm{l} \\
\text { Lymphocyte } \times 10^{3} / \mu \mathrm{l} \\
\text { Eosinophils } \times 10^{3} / \mu \mathrm{l} \\
\text { TLC } \times 10^{3} / \mu \mathrm{l} \\
\text { CRP }(\mathrm{mg} / \mathrm{L}) \\
\text { Ferritin }(\mathrm{ng} / \mathrm{ml}) \\
\text { D-dimer }(\mathrm{mg} / \mathrm{L}) \\
\end{array}$ & $\begin{array}{c}\text { Negative } 3(1.9 \%) \\
\text { Positive } 159(98.1 \%) \\
\text { Mean } \pm \text { SD: } 12.38 \pm 2.05 \\
\text { Range: } 6.9-17.1 \\
\text { Mean: } \pm 230.81 \pm 98.33 \\
\text { Range: } 8-546 \\
\text { Median (IQR): } 1.4(0.77-2.18) \\
\text { Range: } 0.12-7.58 \\
\text { Median (IQR): } 0.03(0-0.07) \\
\text { Range: } 0-1.1 \\
\text { Median (IQR): } 6.73(4.8-9) \\
\text { Range: } 0.8-29.7 \\
\text { Median (IQR): } 15.5(5-48) \\
\text { Range: } 1-164 \\
\text { Median (IQR): } 250(60-717) \\
\text { Range: } 1-2200 \\
\text { Median (IQR): } 0.63(0.2-1.7) \\
\text { Range: } 0.01-10 \\
\end{array}$ \\
\hline Fate & $\begin{array}{c}\text { ICU } \\
\text { Death } \\
\text { Days of hospital stay }\end{array}$ & $\begin{array}{c}45(27.8 \%) \\
10(6.2 \%) \\
\text { Median (IQR): } 10(6-13) \\
\text { Range: } 2-51 \\
\end{array}$ \\
\hline Chest CT & $\begin{array}{c}\text { Free } \\
\text { Unilateral ground glass opacities } \\
\text { Bilateral ground glass opacities } \\
\text { Bilateral ground glass opacities + pneumonia }\end{array}$ & $\begin{aligned} & 63(38.9 \%) \\
& 5(3.1 \%) \\
& 91(56.2 \%) \\
& 3(1.9 \%) \\
&\end{aligned}$ \\
\hline
\end{tabular}

DM, diabetes mellites; HTN, hypertension; CLD, chronic liver disease; CKD, chronic kidney disease; CORADS, COVID-19 Reporting and Data System; HB, hemoglobin; PLT, platelets; CRP, C-reactive protein; TLC, total leucocytic count; ICU, intensive care unit; PCR, polymerase chain reaction. $P$-value $<0.05$ was considered significant. 
Normal reference ranges: absolute eosinophil count: $0.1-1 \times 10^{3} / \mu l$; absolute lymphocyte count: $1.5-4 \times 10^{3} / \mu l$; CRP: $0.5 \mathrm{mg} / \mathrm{L}$; D dimer: up to $0.5 \mathrm{mg} / \mathrm{L}$; Ferritin: male 21.8-274.6ng/ml, female 4.6-204ng/ml; HB: male 13 $17 \mathrm{gm} / \mathrm{dl}$, female $12-15 \mathrm{gm} / \mathrm{dl} ; \boldsymbol{P L T}: 150-450 \times 10^{3} / \mu \mathrm{l} ; \boldsymbol{T L C}: 4-10 \times 10^{3} / \mu \mathrm{l}$

Table (2): Comparison of patients' characteristics (demographic and clinical data, comorbidities, and drug therapy) according to the total leucocytic count (TLC).

\begin{tabular}{|c|c|c|c|c|}
\hline \multirow{2}{*}{\multicolumn{2}{|c|}{ Variable }} & \multicolumn{2}{|c|}{ TLC } & \multirow{2}{*}{ P-value } \\
\hline & & Median (IQR) & Range & \\
\hline \multicolumn{5}{|c|}{ (A)Demographic and clinical } \\
\hline Sex & $\begin{array}{c}\text { Female } \\
\text { Male }\end{array}$ & $\begin{array}{l}6.80(4.81-9.30) \\
6.67(4.80-8.91)\end{array}$ & $\begin{array}{l}0.80-27.00 \\
1.02-29.70\end{array}$ & 0.585 \\
\hline Severity & $\begin{array}{c}\text { Mild } \\
\text { Moderate } \\
\text { Severe } \\
\end{array}$ & $\begin{array}{c}5.80(4.80-7.67) \\
6.90(4.99-9.30) \\
9.00(4.80-17.00)\end{array}$ & $\begin{array}{l}2.20-25.00 \\
2.12-29.70 \\
0.80-27.00 \\
\end{array}$ & 0.019 \\
\hline ICU & $\begin{array}{l}\text { Negative } \\
\text { Positive }\end{array}$ & $\begin{array}{c}6.00(4.80-7.90) \\
8.00(6.00-16.70)\end{array}$ & $\begin{array}{l}1.02-25.00 \\
0.80-29.70\end{array}$ & $\leq 0.001$ \\
\hline Out come & $\begin{array}{l}\text { Died } \\
\text { Alive }\end{array}$ & $\begin{array}{c}12.65(4.52-19.00) \\
6.65(4.80-8.75)\end{array}$ & $\begin{array}{l}0.80-27.00 \\
1.02-29.70\end{array}$ & 0.125 \\
\hline \multicolumn{5}{|c|}{ (B) Comorbidities } \\
\hline SMOKING & $\begin{array}{l}\text { Non-smoker } \\
\text { Smoker }\end{array}$ & $\begin{array}{l}6.90(4.81-9.30) \\
4.99(4.52-6.30)\end{array}$ & $\begin{array}{l}0.80-29.70 \\
1.02-14.00\end{array}$ & 0.065 \\
\hline DM & $\begin{array}{l}\text { Negative } \\
\text { Positive }\end{array}$ & $\begin{array}{c}6.30(4.79-8.00) \\
8.00(6.90-13.00)\end{array}$ & $\begin{array}{l}0.80-27.00 \\
2.36-29.70\end{array}$ & 0.002 \\
\hline HTN & $\begin{array}{l}\text { Negative } \\
\text { Positive }\end{array}$ & $\begin{array}{c}6.59(4.80-8.00) \\
7.05(4.80-11.00)\end{array}$ & $\begin{array}{l}0.80-27.00 \\
1.02-29.70\end{array}$ & 0.201 \\
\hline CLD & $\begin{array}{l}\text { Negative } \\
\text { Positive }\end{array}$ & $\begin{array}{c}6.70(4.80-8.81) \\
25.00(13.00-27.00)\end{array}$ & $\begin{array}{c}0.80-29.70 \\
13.00-27.00\end{array}$ & 0.008 \\
\hline CKD & $\begin{array}{c}\text { Negative } \\
\text { Positive } \\
\text { On dialysis }\end{array}$ & $\begin{array}{c}6.75(4.80-8.81) \\
5.90(4.60-11.65) \\
5.58(3.95-7.20)\end{array}$ & $\begin{array}{c}0.80-29.70 \\
2.97-27.00 \\
3.95-7.20\end{array}$ & 0.809 \\
\hline \multicolumn{5}{|c|}{ (C) Drug therapy } \\
\hline Plaquenil & $\begin{array}{l}\text { Negative } \\
\text { Positive }\end{array}$ & $\begin{array}{c}8.00(5.90-15.20) \\
6.34(4.80-8.00)\end{array}$ & $\begin{array}{l}0.80-27.00 \\
1.02-29.70\end{array}$ & 0.002 \\
\hline Clexane & $\begin{array}{l}\text { Negative } \\
\text { Positive }\end{array}$ & $\begin{array}{l}6.50(4.80-8.80) \\
6.75(4.81-9.00)\end{array}$ & $\begin{array}{l}4.80-27.00 \\
0.80-29.70\end{array}$ & 0.804 \\
\hline Tamiflu & $\begin{array}{l}\text { Negative } \\
\text { Positive }\end{array}$ & $\begin{array}{l}7.00(4.99-9.75) \\
5.45(4.80-7.20)\end{array}$ & $\begin{array}{l}0.80-29.70 \\
2.40-17.00\end{array}$ & 0.075 \\
\hline Avigan & $\begin{array}{l}\text { Negative } \\
\text { Positive }\end{array}$ & $\begin{array}{l}6.70(4.80-9.00) \\
7.81(7.81-7.81)\end{array}$ & $\begin{array}{c}0.80-29.70 \\
7.81-7.81\end{array}$ & 0.571 \\
\hline Remdesivir & $\begin{array}{l}\text { Negative } \\
\text { Positive }\end{array}$ & $\begin{array}{l}6.70(4.80-9.00) \\
8.00(7.25-9.00)\end{array}$ & $\begin{array}{l}0.80-29.70 \\
6.50-10.00\end{array}$ & 0.248 \\
\hline Steroids & $\begin{array}{l}\text { Negative } \\
\text { Positive }\end{array}$ & $\begin{array}{c}5.80(4.80-7.67) \\
7.10(4.81-10.11)\end{array}$ & $\begin{array}{l}2.20-25.00 \\
0.80-29.70\end{array}$ & 0.039 \\
\hline Actemra & $\begin{array}{l}\text { Negative } \\
\text { Positive }\end{array}$ & $\begin{array}{c}6.70(4.80-8.84) \\
6.89(4.80-12.77)\end{array}$ & $\begin{array}{l}0.80-27.00 \\
2.36-29.70\end{array}$ & 0.315 \\
\hline
\end{tabular}

DM, diabetes mellites; HTN, hypertension; $C L D$, chronic liver disease; $C K D$, chronic kidney disease; ICU, intensive care unit; IQR, interquartile range. $P$-value $<0.05$ was considered significant. 
Table (3): Comparison of patients' characteristics (demographic and clinical data, comorbidities, and drug therapy) according to the lymphocyte count.

\begin{tabular}{|c|c|c|c|c|}
\hline \multirow{2}{*}{\multicolumn{2}{|c|}{ Variable }} & \multicolumn{2}{|c|}{ Lymphocytes } & \multirow{2}{*}{ P-value } \\
\hline & & Median (IQR) & Range & \\
\hline \multicolumn{5}{|c|}{ (A)Demographic and clinical } \\
\hline Sex & $\begin{array}{c}\text { Female } \\
\text { Male }\end{array}$ & $\begin{array}{l}1.59(0.98-2.17) \\
1.37(0.70-2.24)\end{array}$ & $\begin{array}{l}0.36-7.58 \\
0.12-4.60 \\
\end{array}$ & 0.518 \\
\hline Severity & $\begin{array}{c}\text { Mild } \\
\text { Moderate } \\
\text { Severe }\end{array}$ & $\begin{array}{l}1.78(1.28-2.54) \\
1.17(0.70-2.00) \\
1.15(0.64-1.92)\end{array}$ & $\begin{array}{l}0.45-4.20 \\
0.12-4.96 \\
0.28-7.58 \\
\end{array}$ & 0.003 \\
\hline ICU & $\begin{array}{l}\text { Negative } \\
\text { Positive }\end{array}$ & $\begin{array}{l}1.70(1.14-2.34) \\
0.80(0.58-1.60)\end{array}$ & $\begin{array}{l}0.28-4.96 \\
0.12-7.58 \\
\end{array}$ & $\leq 0.001$ \\
\hline Out come & $\begin{array}{l}\text { Died } \\
\text { Alive }\end{array}$ & $\begin{array}{l}1.37(0.72-2.11) \\
1.50(0.77-2.18)\end{array}$ & $\begin{array}{l}0.64-7.58 \\
0.12-4.96\end{array}$ & 0.997 \\
\hline \multicolumn{5}{|c|}{ (B)Comorbidities } \\
\hline SMOKING & $\begin{array}{c}\text { Non-smoker } \\
\text { Smoker }\end{array}$ & $\begin{array}{l}1.40(0.75-2.13) \\
1.85(1.14-2.90)\end{array}$ & $\begin{array}{l}0.12-7.58 \\
0.28-4.00\end{array}$ & 0.367 \\
\hline DM & $\begin{array}{l}\text { Negative } \\
\text { Positive }\end{array}$ & $\begin{array}{l}1.55(1.00-2.24) \\
0.97(0.64-1.92)\end{array}$ & $\begin{array}{l}0.12-7.58 \\
0.20-4.96 \\
\end{array}$ & 0.058 \\
\hline HTN & $\begin{array}{l}\text { Negative } \\
\text { Positive }\end{array}$ & $\begin{array}{l}1.60(0.90-2.24) \\
1.14(0.70-1.92)\end{array}$ & $\begin{array}{l}0.12-7.58 \\
0.20-3.50\end{array}$ & 0.040 \\
\hline CLD & $\begin{array}{l}\text { Negative } \\
\text { Positive }\end{array}$ & $\begin{array}{l}1.50(0.80-2.18) \\
0.64(0.40-0.70)\end{array}$ & $\begin{array}{l}0.12-7.58 \\
0.40-0.70 \\
\end{array}$ & 0.028 \\
\hline CKD & $\begin{array}{l}\text { Negative } \\
\text { Positive } \\
\text { On dialysis }\end{array}$ & $\begin{array}{l}1.58(0.77-2.30) \\
1.10(0.55-1.40) \\
0.73(0.70-0.75)\end{array}$ & $\begin{array}{l}0.12-7.58 \\
0.36-2.89 \\
0.70-0.75 \\
\end{array}$ & 0.077 \\
\hline \multicolumn{5}{|c|}{ (C)Drug therapy } \\
\hline Plaquenil & $\begin{array}{l}\text { Negative } \\
\text { Positive }\end{array}$ & $\begin{array}{l}1.26(0.64-1.92) \\
1.50(0.99-2.30)\end{array}$ & $\begin{array}{l}0.12-7.58 \\
0.20-4.96\end{array}$ & 0.156 \\
\hline Clexane & $\begin{array}{l}\text { Negative } \\
\text { Positive }\end{array}$ & $\begin{array}{l}1.15(0.65-1.66) \\
1.50(0.77-2.24)\end{array}$ & $\begin{array}{l}0.40-2.16 \\
0.12-7.58\end{array}$ & 0.210 \\
\hline Tamiflu & $\begin{array}{l}\text { Negative } \\
\text { Positive }\end{array}$ & $\begin{array}{l}1.35(0.73-2.21) \\
1.59(1.10-2.13)\end{array}$ & $\begin{array}{l}0.12-7.58 \\
0.60-4.60\end{array}$ & 0.349 \\
\hline Avigan & $\begin{array}{l}\text { Negative } \\
\text { Positive }\end{array}$ & $\begin{array}{l}1.40(0.76-2.17) \\
2.18(2.18-2.18)\end{array}$ & $\begin{array}{l}0.12-7.58 \\
2.18-2.18\end{array}$ & 0.383 \\
\hline Remdesivir & $\begin{array}{l}\text { Negative } \\
\text { Positive }\end{array}$ & $\begin{array}{l}1.40(0.77-2.18) \\
1.87(0.74-2.02)\end{array}$ & $\begin{array}{l}0.12-7.58 \\
0.74-2.02\end{array}$ & 0.873 \\
\hline Steroids & $\begin{array}{l}\text { Negative } \\
\text { Positive }\end{array}$ & $\begin{array}{l}1.78(1.28-2.54) \\
1.17(0.70-2.00)\end{array}$ & $\begin{array}{l}0.45-4.20 \\
0.12-7.58 \\
\end{array}$ & 0.001 \\
\hline Actemra & $\begin{array}{l}\text { Negative } \\
\text { Positive }\end{array}$ & $\begin{array}{l}1.58(0.86-2.30) \\
1.01(0.74-1.30)\end{array}$ & $\begin{array}{l}0.12-7.58 \\
0.20-2.00\end{array}$ & 0.014 \\
\hline
\end{tabular}

DM, diabetes mellites; HTN, hypertension; $C L D$, chronic liver disease; $C K D$, chronic kidney disease; ICU, intensive care unit; IQR, interquartile range. $P$-value $<0.05$ was considered significant. 
Table (4): Comparison of patients' characteristics (demographic and clinical data, comorbidities, and drug therapy) according to the eosinophil count.

\begin{tabular}{|c|c|c|c|c|}
\hline \multirow{2}{*}{\multicolumn{2}{|c|}{ Variable }} & \multicolumn{2}{|c|}{ Eosinophils } & \multirow{2}{*}{ P-value } \\
\hline & & \multirow[t]{2}{*}{ Median (IQR) } & \multirow[t]{2}{*}{ Range } & \\
\hline (A)Demogr & clinical & & & \\
\hline Sex & $\begin{array}{c}\text { Female } \\
\text { Male }\end{array}$ & $\begin{array}{l}0.04(0.00-0.07) \\
0.03(0.00-0.06)\end{array}$ & $\begin{array}{l}0.00-1.10 \\
0.00-0.80\end{array}$ & 0.607 \\
\hline Severity & $\begin{array}{c}\text { Mild } \\
\text { Moderate } \\
\text { Severe }\end{array}$ & $\begin{array}{l}0.03(0.00-0.07) \\
0.04(0.00-0.09) \\
0.03(0.00-0.06)\end{array}$ & $\begin{array}{l}0.00-0.50 \\
0.00-1.10 \\
0.00-0.26\end{array}$ & 0.649 \\
\hline ICU & $\begin{array}{l}\text { Negative } \\
\text { Positive }\end{array}$ & $\begin{array}{l}0.03(0.00-0.07) \\
0.04(0.00-0.07)\end{array}$ & $\begin{array}{l}0.00-0.80 \\
0.00-1.10\end{array}$ & 0.551 \\
\hline Out come & $\begin{array}{l}\text { Died } \\
\text { Alive }\end{array}$ & $\begin{array}{l}0.04(0.00-0.06) \\
0.03(0.00-0.07)\end{array}$ & $\begin{array}{l}0.00-0.22 \\
0.00-1.10\end{array}$ & 0.994 \\
\hline \multicolumn{2}{|c|}{ (B)comorbidities } & & & \\
\hline Smoking & $\begin{array}{c}\text { Non-smoker } \\
\text { Smoker }\end{array}$ & $\begin{array}{l}0.03(0.00-0.07) \\
0.04(0.00-0.12)\end{array}$ & $\begin{array}{l}0.00-1.10 \\
0.00-0.36\end{array}$ & 0.757 \\
\hline DM & $\begin{array}{l}\text { Negative } \\
\text { Positive }\end{array}$ & $\begin{array}{l}0.03(0.00-0.07) \\
0.05(0.00-0.08)\end{array}$ & $\begin{array}{l}0.00-0.80 \\
0.00-1.10\end{array}$ & 0.441 \\
\hline HTN & $\begin{array}{l}\text { Negative } \\
\text { Positive }\end{array}$ & $\begin{array}{l}0.03(0.00-0.08) \\
0.04(0.00-0.06)\end{array}$ & $\begin{array}{l}0.00-0.80 \\
0.00-1.10\end{array}$ & 0.742 \\
\hline CLD & $\begin{array}{l}\text { Negative } \\
\text { Positive }\end{array}$ & $\begin{array}{l}0.03(0.00-0.07) \\
0.00(0.00-0.22)\end{array}$ & $\begin{array}{l}0.00-1.10 \\
0.00-0.22\end{array}$ & 0.743 \\
\hline CKD & $\begin{array}{c}\text { Negative } \\
\text { Positive } \\
\text { On dialysis } \\
\end{array}$ & $\begin{array}{l}0.03(0.00-0.07) \\
0.02(0.00-0.05) \\
0.01(0.00-0.02)\end{array}$ & $\begin{array}{l}0.00-1.10 \\
0.00-0.50 \\
0.00-0.02 \\
\end{array}$ & 0.461 \\
\hline \multicolumn{2}{|c|}{ (C)Drug therapy } & & & \\
\hline Plaquenil & $\begin{array}{l}\text { Negative } \\
\text { Positive }\end{array}$ & $\begin{array}{l}0.04(0.03-0.06) \\
0.03(0.00-0.08)\end{array}$ & $\begin{array}{l}0.00-0.22 \\
0.00-1.10\end{array}$ & 0.437 \\
\hline Clexane & $\begin{array}{l}\text { Negative } \\
\text { Positive }\end{array}$ & $\begin{array}{l}0.00(0.00-0.00) \\
0.04(0.00-0.07)\end{array}$ & $\begin{array}{l}0.00-0.09 \\
0.00-1.10\end{array}$ & 0.039 \\
\hline Tamiflu & $\begin{array}{l}\text { Negative } \\
\text { Positive }\end{array}$ & $\begin{array}{l}0.04(0.00-0.08) \\
0.03(0.00-0.06)\end{array}$ & $\begin{array}{l}0.00-1.10 \\
0.00-0.80\end{array}$ & 0.430 \\
\hline Avigan & $\begin{array}{l}\text { Negative } \\
\text { Positive }\end{array}$ & $\begin{array}{l}0.03(0.00-0.07) \\
0.04(0.04-0.04)\end{array}$ & $\begin{array}{l}0.00-1.10 \\
0.04-0.04\end{array}$ & 0.894 \\
\hline Remdesivir & $\begin{array}{l}\text { Negative } \\
\text { Positive }\end{array}$ & $\begin{array}{l}0.03(0.00-0.07) \\
0.05(0.02-0.08)\end{array}$ & $\begin{array}{l}0.00-1.10 \\
0.00-0.09\end{array}$ & 0.679 \\
\hline Steroids & $\begin{array}{l}\text { Negative } \\
\text { Positive }\end{array}$ & $\begin{array}{l}0.03(0.00-0.07) \\
0.04(0.00-0.08)\end{array}$ & $\begin{array}{l}0.00-0.50 \\
0.00-1.10\end{array}$ & 0.663 \\
\hline Actemra & $\begin{array}{l}\text { Negative } \\
\text { Positive }\end{array}$ & $\begin{array}{l}0.03(0.00-0.07) \\
0.04(0.00-0.09)\end{array}$ & $\begin{array}{l}0.00-0.80 \\
0.00-1.10\end{array}$ & 0.974 \\
\hline
\end{tabular}

DM, diabetes mellites; HTN, hypertension; $C L D$, chronic liver disease; $C K D$, chronic kidney disease; ICU, intensive care unit; IQR, interquartile range. $P$-value $<0.05$ was considered significant. 
Table (5): Correlations of TLC, lymphocyte and eosinophil counts with different laboratory test results, age of patients, COVID-19 CO-RADS classification and length of hospital stay in all studied cases.

\begin{tabular}{|c|c|c|c|c|c|c|}
\hline \multirow{2}{*}{ Variable } & \multicolumn{2}{|c|}{ Lymphocytes } & \multicolumn{2}{|c|}{ Eosinophils } & \multicolumn{2}{|c|}{ TLC } \\
\hline & $\mathbf{r}$ & P-value & $\mathbf{r}$ & P-value & $\mathbf{r}$ & P-value \\
\hline Lymphocytes & - & - & 0.111 & 0.167 & 0.022 & 0.785 \\
\hline Eosinophils & 0.111 & 0.167 & - & - & 0.077 & 0.332 \\
\hline TLC & 0.022 & 0.785 & 0.077 & 0.332 & - & - \\
\hline Age & -0.187 & 0.019 & 0.140 & 0.076 & 0.078 & 0.321 \\
\hline CO-RADS & -0.198 & 0.013 & 0.046 & 0.561 & 0.161 & 0.041 \\
\hline HB & $\mathbf{0 . 3 3 1}$ & $\leq 0.001$ & 0.047 & 0.555 & -0.069 & 0.382 \\
\hline PLT & 0.130 & 0.108 & 0.032 & 0.684 & 0.211 & 0.007 \\
\hline CRP & -0.218 & 0.006 & 0.035 & 0.658 & 0.324 & $\leq 0.001$ \\
\hline Ferritin & -0.314 & $\leq 0.001$ & 0.030 & 0.705 & 0.232 & 0.003 \\
\hline D-dimer & -0.260 & 0.001 & -0.006 & 0.938 & 0.279 & $\leq 0.001$ \\
\hline Hospital stay (days) & 0.036 & 0.655 & 0.019 & 0.808 & 0.005 & 0.950 \\
\hline
\end{tabular}

TLC, total leucocytic count; CO-RADS, COVID-19 Reporting and Data System; HB, hemoglobin; PLT, platelets; CRP, C-reactive protein; $r$, correlation coefficient. P-value $<0.05$ was considered significant.

\section{DISCUSSION}

This study aimed to correlate total blood counts, lymphocytic, and eosinophilic counts with the COVID-19 severity, hoping to predict future sequelae of the disease, thus allowing clinicians to make proper management strategies as well early as possible.

Total leucocytic count (TLC) and differential counts showed no statistically significant difference between males and females in our study. In contrast, Haitao et al. [8] reported that men are consistently more infected by SARS$\mathrm{CoV}-2$ and more prone to its severe outcomes and higher mortality rates due to gender-related behaviors, social and comorbid factors, as well as genetic and hormonal factors.

Our study revealed that diabetic patients with COVID-19 had statistically significant higher TLC and that hypertensive patients had statistically significant lower lymphocyte counts. Similarly, Anurag et al. [9] observed that both neutrophil lymphocytic ratio (NLR) and neutrophil monocytic ratio (NMR) were indicative of severe COVID-19 infection, and both were significantly increased in diabetic patients. In contrast, NLR alone was significantly increased in hypertensive patients. They explained their findings by the dysregulated glucose metabolism in diabetics, which can lead to dysfunctional immunity and hypercoagulability, and angiotensin-converting enzyme-2 (ACE-2) receptor inhibitors and blockers used in the treatment of hypertensives that might increase ACE-2 receptor expression, facilitating SARS-CoV-2 entry to cells.

Our work showed that COVID-19 patients with chronic liver disease (CLD) had statistically significant higher TLC and lower lymphocyte counts. Cerbu et al. [10] indicated that immunocompromised COVID-19 patients with CLD had a more severe disease course, comorbidities, and outcomes; also, those patients had abnormally high aminotransferases, LDH, CRP and coagulation tests and low albumin.

We concluded that eosinophil counts did not show any significant changes with any comorbidities associated with COVID-19 patients. However, Rosenberg and Foster [4] have listed some complications associated with eosinophilia in COVID-19 patients, including isolated pulmonary eosinophilic vasculitis, acute eosinophilic pneumonia, fatal eosinophilic myocarditis, eosinophilic granulomatosis, with polyangiitis.

Regarding COVID-19 disease severity in our patients, the TLC significantly increased, and the lymphocyte counts significantly decreased with more severe disease. Selim [11] stated that the COVID-19 virus attaches to ACE2 receptors on type 2 pneumocytes, lymphocytes, and renal epithelial cells, causing direct damage to these target cells. The virus also causes a cytokine storm, leading to acute respiratory distress syndrome and multiple organ failure. $\mathrm{He}$ confirmed that neutrophilia and/or lymphocytopenia related to the cytokine storm 
were associated with more severe cases of COVID-19 disease and poor prognosis.

Mao et al. [12] also found that critically ill patients had persistent leukocytosis, neutrophilia, monocytosis, lymphocytopenia, eosinopenia, and anemia. They suggested that the virus may directly damage the hematopoietic and immune system, with subsequent hypoplastic bone marrow and lymphopenia.

On the other hand, eosinophil counts observed in our study showed no significant changes with the severity of COVID-19. Koenderman et al. [13] have stated that although eosinopenia is a common finding in COVID-19 at hospital presentation, yet it is not specific for SARSCoV-2 infection neither related to its severity. They realized that patients with negative SARSCoV-2 PCR and suffering from either bacterial or another viral infection also showed less pronounced eosinopenia and assumed that acute systemic inflammation could lead to eosinopenia irrespective of the causative agent.

Also, Lippi et al. [14] agreed with our results as they found only a marginally significant difference in the count of eosinophils in severe COVID-19 patients compared to milder cases. Therefore, they doubted the efficiency of eosinophil count for predicting severe COVID19 disease because of the biological variation in eosinophil counts and the current technical drawbacks in measuring eosinophils in whole blood.

Qian et al. [15] as well reported that the relation between eosinopenia and COVID-19 was questionable, attributing eosinopenia to the sequestration of eosinophils by chemokines into the local inflammatory sites or suppression in the bone marrow.

While Rosenberg and Foster [4] have reported a different finding as they concluded that eosinopenia could facilitate both the diagnosis and prognosis of severe COVID-19, but it is neither definitive nor pathognomonic for COVID-19. They also reported some algorithms which predict COVID-19 severity and include eosinophil counts like the risk stratification score (COVID-19-REAL) and PAthRIS score.

In our study, eosinophil counts were significantly increased in patients treated with Clexane. Similarly, Ari et al. [16] have linked high eosinophil count to lower anti-factor Xa activity in patients with COVID-19 receiving low molecular weight heparin (LMWH). That is why they suggested that eosinophil counts can be used as an alarm to adjust the anticoagulation dose.

Moreover, our study showed that TLC was significantly decreased in patients treated with Plaquenil. Sames et al. [17] listed that rare side effects of hydroxychloroquine (Plaquenil) included leukopenia due to agranulocytosis and bone marrow suppression. On the other hand, our study showed that TLC was significantly increased in patients treated with steroids. Eljaaly et al. [18] also stated that glucocorticoids could cause leukocytosis, neutrophilia, and lymphopenia.

In the same context, our results showed a significant decrease in lymphocyte counts with treatment with Actemra or tocilizumab. Keske et al. [19] stated that the early administration of tocilizumab in COVID-19 disease improved survival and decreased the hospitalization duration and the need for oxygen support. It is an anti-IL-6 receptor monoclonal antibody that inhibits signal transduction and suppresses the cytokine storm. The earliest alarming signs that permit Actemra use include elevated CRP, IL-6, and decreased \% lymphocytes, which improve upon its administration.

In our study, eosinophil counts did not show significant changes either in patients admitted to the ICU or with the fate of the patients. In accordance, Le Borgne et al. [20] concluded that extreme eosinopenia was predictive of COVID19 severity but not mortality. While a study by Yan et al. [1] highlighted that progressively worsening eosinopenia was a sign of critical disease and higher mortality because eosinopenia correlated with biomarkers of coagulopathy and kidney and liver damage. Also, another study by Nair et al. [21] found that patients with eosinophilia had a lower CRP level, milder clinical course, and better disease outcomes indicating a protective role of eosinophils against severe inflammation via an inhibitory mechanism.

In all patients in our study, TLC showed significant positive correlations with COVID-19 severity, CO-RADS chest CT imaging classification, CRP, ferritin, and D-dimer levels. Anurag et al. [9] also realized that increased TLC, neutrophilia, lymphopenia, high NLR, and high NMR were associated with COVID-19 severity. Zhu et al. [22] specified that TLC count 
$\left(\geq 6.16 \times 10^{9} / \mathrm{L}\right)$ should receive more caution during treatment because TLC count at admission was significantly correlated with death in COVID-19 patients. Similarly, a study by Amano et al. [23] showed that Chest CT imaging had a vital diagnostic role in COVID-19 due to its high sensitivity and correlation with disease severity, together with increased CRP, ferritin, D-dimer, and lymphopenia.

Our work showed that lymphocyte count had a significant positive correlation with hemoglobin level. Zhang et al. [24] also reported that lymphocytes and hemoglobin decreased gradually with disease progression. They explained lymphopenia by direct viral infection to lymphocytes, direct viral destruction of lymphatic organs, inflammatory cytokines release like tumor necrosis factor-alpha and IL-6, or lymphocytic inhibition by metabolic products. They also explained the decrease in hemoglobin by viral adherence to hematopoietic cells through the ACE 2 receptor releasing endotoxins and destroying the hematopoietic stem/progenitor cells.

We additionally found that lymphocytes had significant negative correlations with age, COVID-19 CO-RADS classification, CRP, ferritin, and d-dimer levels. Zhang et al. [24] as well found that older age was accompanied by more severe disease, probably due to the weakening of the body's immune system or the presence of underlying comorbidity. Hashem et al. [25] investigated the prognostic value of the available laboratory biomarkers and concluded that lymphopenia together with anemia and leukocytosis and inflammation-related proteins like CRP, ferritin, D-dimer, procalcitonin, and IL-6 played independent roles in identifying severe cases with poor outcomes.

Finally, our work revealed no significant correlations between eosinophil count and any of the laboratory test results. Karimi Shahri et al. [26] have also stated that although eosinophils could have an antiviral role yet, it remains controversial when it concerns COVID-19. And although eosinopenia has a bad prognostic value, its actual involvement in the inflammatory process of infectious diseases is yet to be established. Eosinopenia in SARS-CoV-2 infection has been explained by eosinophils migration, like lymphocytes, into the pulmonary parenchyma, with a subsequent decrease in the circulating eosinophil count. However, their role against COVID-19 infection is not pathognomonic.

\section{CONCLUSIONS:}

In our study, the eosinophil count showed no significant correlation with the disease severity or with the other typical biomarkers of COVID19 disease, while lymphopenia was persistently a poor prognostic marker. Until the analytical imprecision and the functional sensitivity of the modern hematological analyzers is improved, depending on the eosinophil count for diagnosis, prognosis, and treatment of COVID-19 would hence remain questionable.

Funding sources: This research did not receive any specific grant from funding agencies in the public, commercial, or not-for-profit sectors.

Acknowledgement: None.

Conflicts of interest: The authors declare that there is no conflict of interest regarding the publication of this article.

Consent for publication: Not applicable.

Availability of data and materials: All data needed to support the current findings will be available upon request.

ABBREVIATIONS:

ACE-2: Angiotensin-converting enzyme-2

CBC: Complete blood count

CLD: Chronic liver disease

COVID-19: Coronavirus disease 2019

IL: Interleukin

NLR: Neutrophil lymphocytic ratio

NMR: Neutrophil monocytic ratio

SARS-CoV-2: Severe Acute Respiratory Syndrome Coronavirus 2

\section{ETHICAL CONSIDERATIONS}

The study followed the Declaration of Helsinki's ethical research criteria. The Ain Shams University Faculty of Medicine Research Ethics Committee approved the current study procedure. All patient data was kept private, only used for the study. 


\section{HIGHLIGHTS:}

- The total leucocytic count and absolute lymphocyte count may serve as predictive biomarkers for COVID-19 progression.

- Total leucocytic count significantly increases with COVID-19 severity, while the absolute lymphocyte count significantly decreases

- Absolute eosinophil count does not change significantly with COVID-19 severity

\section{REFERENCES}

1. Yan B, Yang J, Xie Y, Tang X. Relationship between blood eosinophil levels and COVID-19 mortality. World Allergy Organ J. 2021 Mar; 14(3):100521. doi: 10.1016/j.waojou.2021. 100521. Epub 2021 Feb 11. PMID: 33589865; PMCID: PMC7877210.

2. Esnault S, Kelly EA. Essential Mechanisms of Differential Activation of Eosinophils by IL-3 Compared to GM-CSF and IL-5. Crit Rev Immunol. 2016; 36(5):429-444. doi: 10.1615/CritRevImmunol.2017020172. PMID: 28605348 ; PMCID: PMC5586489.

3. Barnes PJ. Inflammatory mediator receptors and asthma. Am Rev Respir Dis. 1987 Jun;135(6 Pt 2): S26-31. doi: 10.1164/arrd.1987.135.6P2.S26. PMID: 2884908.

4. Rosenberg HF, Foster PS. Eosinophils and COVID-19: diagnosis, prognosis, and vaccination strategies. Semin Immunopathol. 2021 Jun; 43(3):383-392. doi: 10.1007/s00281-021-00850-3. Epub 2021 Mar 16. PMID: 33728484; PMCID: PMC7962927.

5. Outh R, Boutin C, Gueudet P, Suzuki M, Saada M, Aumaître H. Eosinopenia $<100 / \mu \mathrm{L}$ as a marker of active COVID-19: An observational prospective study. J Microbiol Immunol Infect. 2021 Feb; 54(1):61-68. doi: 10.1016/j.jmii.2020. 12.005. Epub 2021 Jan 8. PMID: 33468435; PMCID: PMC7792500.

6. World Health Organization. Coronavirus disease (COVID-19) outbreak, https://www.who.int/emereencies/diseases/novelcoronavirus-2019/situation-reports (accessed 11 March 2019).

7. Prokop M, Van Everdingen W, Van Rees Vellinga T, Quarles van Ufford H, Stöger L, et al. CORADS: A Categorical CT Assessment Scheme for Patients Suspected of Having COVID-19 Definition and Evaluation. Radiology. 2020 Aug; 296(2): E97-E104. doi: 10.1148/radiol. 2020201473. PMID: 32339082; PMCID: PMC7233402
8. Haitao T, Vermunt JV, Abeykoon J, Ghamrawi R, Gunaratne M, Jayachandran M, et al. COVID-19 and Sex Differences: Mechanisms and Biomarkers. Mayo Clin Proc. 2020 Oct; 95(10):2189-2203. doi: 10.1016/j.mayocp. 2020.07.024. Epub 2020 Aug 4. PMID: 33012349; PMCID: PMC7402208.

9. Anurag A, Jha PK, Kumar A. Differential white blood cell count in the COVID-19: A crosssectional study of 148 patients. Diabetes Metab Syndr. 2020 Nov-Dec;14(6):2099-2102. doi: 10.1016/j.dsx.2020.10.029. Epub 2020 Nov 2. PMID: 33160224; PMCID: PMC7605785.

10. Cerbu B, Pantea S, Bratosin F, Vidican I, Turaiche M, Frent S, et al. Liver Impairment and Hematological Changes in Patients with Chronic Hepatitis C and COVID-19: A Retrospective Study after One Year of Pandemic. Medicina (Kaunas). 2021 Jun 10; 57(6):597. doi: 10.3390/medicina57060597. PMID: 34200570; PMCID: PMC8226804.

11. Selim S. Leukocyte count in COVID-19: an important consideration. The Egyptian Journal of Bronchology. 2020; 14 (43).

12. Mao J, Dai R, Du RC, Zhu Y, Shui LP, Luo XH. Hematologic changes predict clinical outcome in recovered patients with COVID-19. Ann Hematol. 2021 Mar;100(3):675-689. doi: 10.1007/s00277021-04426-x. Epub 2021 Feb 1. PMID: 33523290; PMCID: PMC7848253.

13. Koenderman L, Siemers MJ, van Aalst C, Bongers SH, Spijkerman R, Bindels BJJ, et al. The Systemic Immune Response in COVID-19 Is Associated with a Shift to Formyl-Peptide Unresponsive Eosinophils. Cells. 2021 May 5;10(5):1109. doi: 10.3390/cells10051109. PMID: 34062964; PMCID: PMC8147959.

14. Lippi G, Sanchis-Gomar F, Henry BM. Response to: Eosinophil count in coronavirus disease 2019: more doubts than answers. QJM. $2021 \mathrm{Feb}$ 18;114(1):70-71. doi: 10.1093/qjmed/hcaa260. PMID: 32877527; PMCID: PMC7499705.

15. Qian GQ, Zhang X, Ma AHY, Yang NB. Response to: Eosinophil count in severe coronavirus disease 2019. QJM. 2020 Jul 1;113(7):513-514. doi: 10.1093/qjmed/hcaa138. PMID: 32315421 ; PMCID: PMC7188165.

16. Ari S, Can V, Demir ÖF, Ari H, Ağca FV, Melek $\mathrm{M}$, et al. Elevated eosinophil count is related with lower anti-factor Xa activity in COVID-19 patients. J Hematop. 2020 Oct 8:1-10. doi: 10.1007/s12308-020-00419-3. Epub ahead of print. PMID: 33046998; PMCID: PMC7541761. 
17. Sames E, Paterson H, Li C. Hydroxychloroquineinduced agranulocytosis in a patient with longterm rheumatoid arthritis. Eur J Rheumatol. 2016 Jun;3(2):91-92. doi: 10.5152/eurjrheum.2015. 0028. Epub 2015 Aug 21. PMID: 27708979; PMCID: PMC5042238.

18. Eljaaly K, Alshehri S, Alshibani M, Katz M. Impact of single-dose systemic glucocorticoids on blood leukocytes in hospitalized adults. Journal of Applied Hematology. 2020; 11(3): 116-121.

19. Keske Ş, Tekin S, Sait B, İrkören P, Kapmaz M, Çimen C, et al. Appropriate use of tocilizumab in COVID-19 infection. Int J Infect Dis. 2020 Oct;99:338-343. doi: 10.1016/j.ijid.2020.07.036. Epub 2020 Jul 26. PMID: 32726724; PMCID: PMC7382959.

20. Le Borgne P, Abensur Vuillaume L, Alamé K, Lefebvre F, Chabrier S, Bérard L, et al. Do Blood Eosinophils Predict in-Hospital Mortality or Severity of Disease in SARS-CoV-2 Infection? A Retrospective Multicenter Study. Microorganisms. 2021 Feb 8;9(2):334. doi: 10.3390/microorganisms9020334. PMID: 33567583; PMCID: PMC7914916.

21. Nair AP, Soliman A, Al Masalamani MA, De Sanctis V, Nashwan AJ, Sasi S, et al. Clinical Outcome of Eosinophilia in Patients with COVID19: A Controlled Study. Acta Biomed. 2020 Nov 10;91(4):e2020165. doi: 10.23750/abm.v91i4. 10564. PMID: 33525219; PMCID: PMC7927494.

22. Zhu B, Feng X, Jiang C, Mi S, Yang L, Zhao Z, et al. Correlation between white blood cell count at admission and mortality in COVID-19 patients: a retrospective study. BMC Infect Dis. 2021 Jun 14;21(1):574. doi: 10.1186/s12879-021-06277-3. PMID: 34126954; PMCID: PMC8202964.

23. Amano Y, Kage H, Tanaka G, Gonoi W, Nakai Y, Kurokawa R, et al. Diagnostic prediction of COVID-19 based on clinical and radiological findings in a relatively low COVID-19 prevalence area. Respir Investig. $2021 \mathrm{Jul}$;59(4):446-453. doi: 10.1016/j.resinv.2021.03.002. Epub 2021 Mar 29. PMID: 33865743; PMCID: PMC8006199.

24. Zhang W, Zhang Z, Ye Y, Luo Y, Pan S, Qi H, et al. Lymphocyte percentage and hemoglobin as a joint parameter for the prediction of severe and nonsevere COVID-19: a preliminary study. Ann Transl Med. 2020 Oct;8(19):1231. doi: 10.21037/atm-20-6001. PMID: 33178763 ; PMCID: PMC7607120.

25. Hashem MK, Khedr EM, Daef E, Hussein A, Mostafa EF, Hassany SM, et al. Prognostic biomarkers in COVID-19 infection: value of anemia, neutrophil-to-lymphocyte ratio, plateletto-lymphocyte ratio, and D-dimer. The Egyptian Journal of Bronchology; 2021; 15(29).

26. Karimi Shahri M, Niazkar HR, Rad F. COVID-19 and hematology findings based on the current evidences: A puzzle with many missing pieces. Int J Lab Hematol. 2021 Apr;43(2):160-168. doi: 10.1111/ijlh.13412. Epub 2020 Dec 2. PMID: 33264492; PMCID: PMC7753300. 